\title{
A modified empirical model for estimating the wetted zone dimensions under drip irrigation
}

\begin{abstract}
Drip irrigation system has become one of the most common irrigation systems especially in arid and semi-arid regions due to its advantages in saving water. One of the most essential considerations in designing these systems is the dimensions of the wetted soil volume under emitters. These dimensions are significant in choosing the proper emitter spacing along the laterals and the suitable distance between laterals. In this study, a modified empirical equations for estimating the horizontal and vertical extend of the wetted zone under surface emitters were suggested. Data from published papers includes different conditions of soil properties and emitter discharge were used in deriving the empirical model using the nonlinear regression. The developed model has high value for coefficient of determination, R2. The results from the developed model were compared with results of other empirical models derived by other researchers. Some statistical criteria were used to evaluate the model performance which are the mean error ME, root mean square error RMSE, and model efficiency EF. The results revealed that the modified model showed good performance in predicting the wetted zone dimensions and it can be used in design and management of drip irrigation systems.
\end{abstract}

Keyword: Drip irrigation; Wetted zone; Empirical model; Wetted radius; Wetted depth 